\title{
FAST CLOSED ORBIT CONTROL IN THE SLS STORAGE RING
}

\author{
M. Böge, M. Dehler, T. Schilcher, V. Schlott, R. Ursic, Paul Scherrer Institute, Switzerland
}

\section{Abstract}

Concepts for a fast orbit feedback in the SLS storage ring are presented. The Singular Value Decomposition (SVD) based orbit correction scheme is supposed to fight orbit oscillations up to $100 \mathrm{~Hz}$ stabilizing the orbit to 1/10th of the beam sigma at the locations of the insertion devices. For an emittance ratio of $1 \%$, this corresponds to $1 \mu \mathrm{m}$ tolerance in the vertical plane. In order to achieve this high accuracy strong restrictions are imposed on the residual noise of the beam position monitoring and the correction system. Theoretical estimates for the feedback gain are made using a simplified feedback model. The proposed layout of the feedback electronics based on the SLS digital BPM system [1] is also described.

\section{ORBIT STABILITY}

The initial orbit stability is determined by three factors:

- The ground motion spectrum

- The girder response

- The orbit response

Measurements of the ground motion spectrum [3] indicate that the amplitudes are well below $100 \mathrm{~nm}$ except for a $27 \mathrm{~Hz} / 300 \mathrm{~nm}$ peak which however could not be reproduced in recent measurements [4]. Assuming a girder response factor of ten for certain eigenmodes the maximum excursion of the elements on the girder is determined to be $3 \mu \mathrm{m}$. Taking into account simulation results for the corresponding orbit response orbit excursions of the electron beam with rms values of up to $15 \mu \mathrm{m}$ [2] at the location of the experiments are expected for the vertical plane. This corresponds to $150 \%$ of the vertical beam sigma for an emittance coupling of 1\% (It should be noted that this coupling factor is somehow arbitrary in the sense that much smaller coupling ratios are achievable utilizing a proper coupling compensation of the lattice [2]). This worst case estimate shows that it is necessary to introduce a fast orbit feedback system which attenuates the oscillations to the desired limit.

In this paper a concept for such a feedback system is presented. It is followed by a description of the actual implementation at the SLS storage ring. Requirements on corrector granularity and BPM noise are defined. Simulations will then show that a fast orbit feedback is capable of attenuating the residual oscillations such that the requirements of the experiments are fulfilled.

\section{FAST ORBIT FEEDBACK}

In general an orbit feedback detects the orbit oscillations induced by incoming ground waves using the beam position monitors around the ring. A lattice dependent well known correlation between monitors and corrector magnets is then used to fight the oscillation (see subsection "Theoretical Concepts"). No information on amplitude, frequency and phase of the distortion is needed for the correction. If the noise source would be exactly known a feed forward would be much more appropriate and much easier to implement. There are two different approaches to implement a feedback. Firstly it could be tried to minimize orbit variations at each experiment individually involving only correctors around the insertion device. This would have the main disadvantage that non locality of the correction would lead to a crosstalk between the local feedbacks which could be extremely difficult to control. A much better alternative represents a global orbit feedback which tries to minimize the orbit variations at all experiments at the same time. Following this approach a global orbit feedback will be adopted at SLS.

\subsection{Theoretical Concepts}

The correlation between correctors and monitors for the linear optics is established by superimposing the monitor reading pattern for every single corrector. The horizontal and vertical plane are treated independently assuming a small betatron coupling. The coefficients of the two resulting correlation matrices also called response matrices can be derived analytically from the machine model or from orbit measurements in the real machine. In the SLS storage ring these matrices have a dimension of $72 \times 72$ corresponding to the 72 correctors per plane built into sextupoles and 72 monitors adjacent to the corresponding sextupoles. To turn this into a correction algorithm it is necessary to invert the matrices in order to get the corrector pattern as a function of a given monitor pattern. If the correlation matrix is a quadratic $n \times n$ matrix and has $n$ independent eigenvectors this is easy to accomplish and one gets a unique solution for the problem. In reality the number of correctors and monitors can be reduced due to monitor failures and magnet saturations in such a way that the matrix is no longer quadratic and the solution is no longer unique.

A very flexible way to handle these scenarios offers the SVD algorithm [5]. This numerically very robust method minimizes the rms orbit and the rms orbit kick at the same time if the number of correctors is larger than the number of monitors while the rms orbit is minimized in the reverse case. Figure 1 depicts the $72 \times 72$ correlation matrix for the vertical plane as derived from the machine model. 


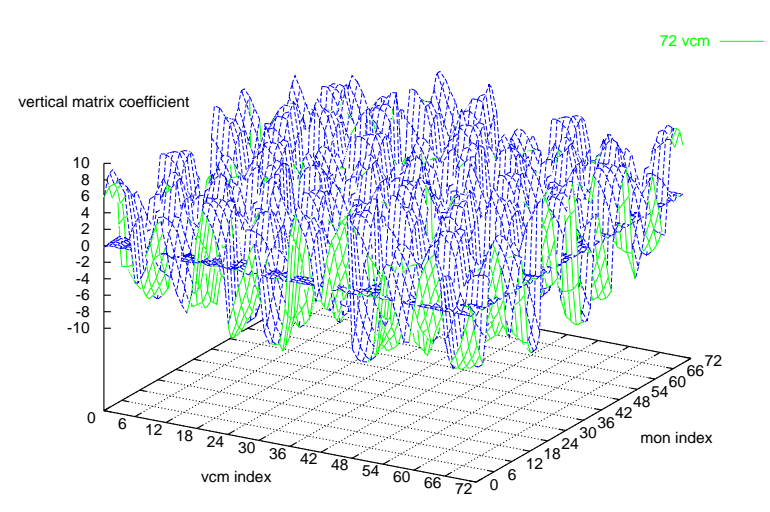

Figure 1: Response matrix for the vertical plane.

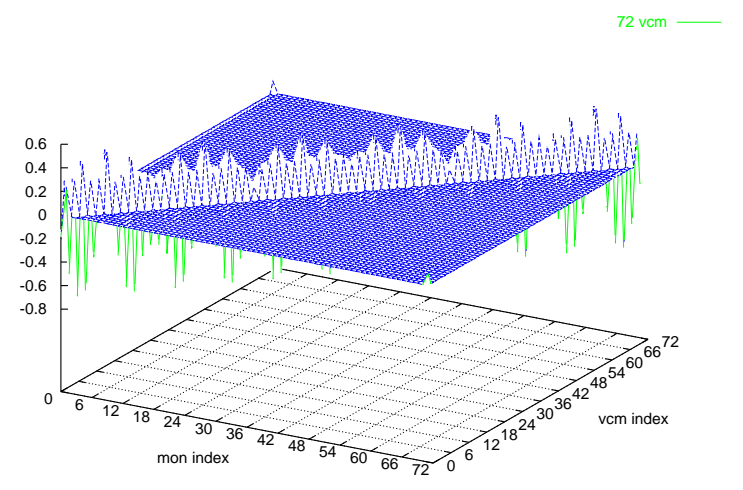

Figure 2: Inverse of the response matrix shown in Figure 1 for the vertical plane.

It should be noted that the coefficients of the matrix are highly correlated. As a result the inverse matrix in Figure 2 has the property that only the diagonal and their adjacent coefficients have significant values. In spite of the fact that the matrix contains the global correlation information of all correctors and monitors the nonzero coefficients gather around the diagonal of the matrix. Thus given a corrector only adjacent monitors determine its value.

This is also true under the condition that the number of correctors used for the orbit correction is reduced. Figure 3 illustrates this for the first row of the nonquadratic "inverse" matrix which corresponds to the first vertical corrector. Successively the total number of vertical correctors ("vcm"'s) is reduced from 72 to 48 and 36 (every third/second corrector is disabled). For the latter case 12 adjacent coefficients are necessary to determine the value of the corrector instead of 3 in the 72 "vcm" case. The same statement holds for the removal of certain ineffective corrector combinations by zeroing the corresponding small weighting factors calculated by SVD. This has a direct influence on the implementation of the feedback.

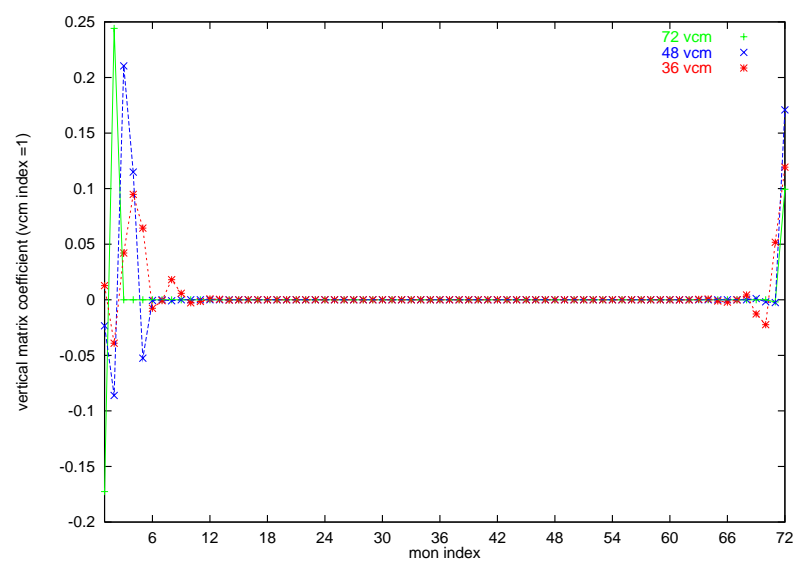

Figure 3: First row of the "inverse" of the response matrix for 72 monitors and 72, 48 and 36 vertical correctors ("vcm"'s).

\subsection{Implementation}

The SLS storage ring is partitioned in 12 sectors containing 6 monitors and 6 correctors per plane. Figure 4 illustrates the partitioning of the inverse response matrix. Corresponding submatrices are distributed to the sectors in order to determine their corrector settings based on their monitor readings. Obviously there must be the possibility to get monitor and matrix information from the adjacent sectors to cover correctors close to the edges of a sector. This can be implemented by means of dedicated links between adjacent sectors. The result is a "leap frog" link structure around the machine (see Figure 4).

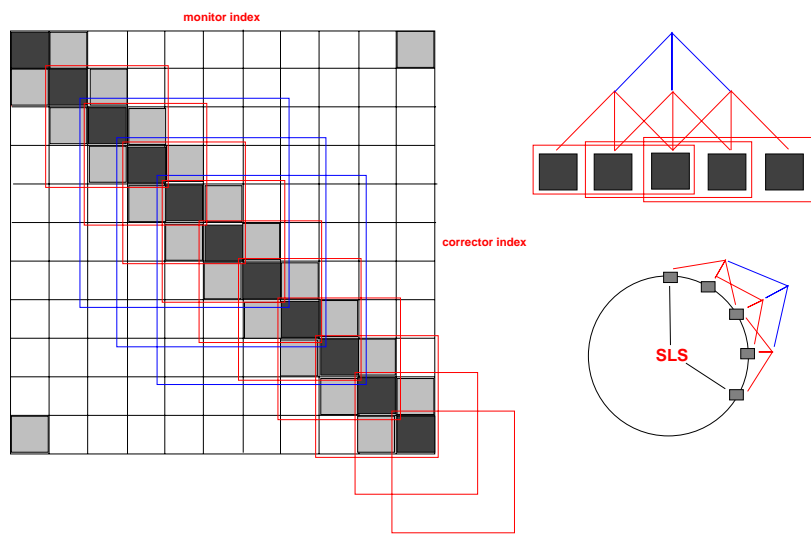

Figure 4: Schematic view of the inverse of the response matrix partitioned into 12 sectors (black boxes) containing 6 monitors and 6 correctors each. The principle layout of the proposed "leap frog" link structure between sectors is shown on the right side.

Figure 5 shows a schematic view of the implementation of the feedback. A central unit named "SVD Engine" performs the SVD based response matrix inversion and distributes the submatrices. All subsequent matrix operations are then performed in parallel by the individual sectors. 


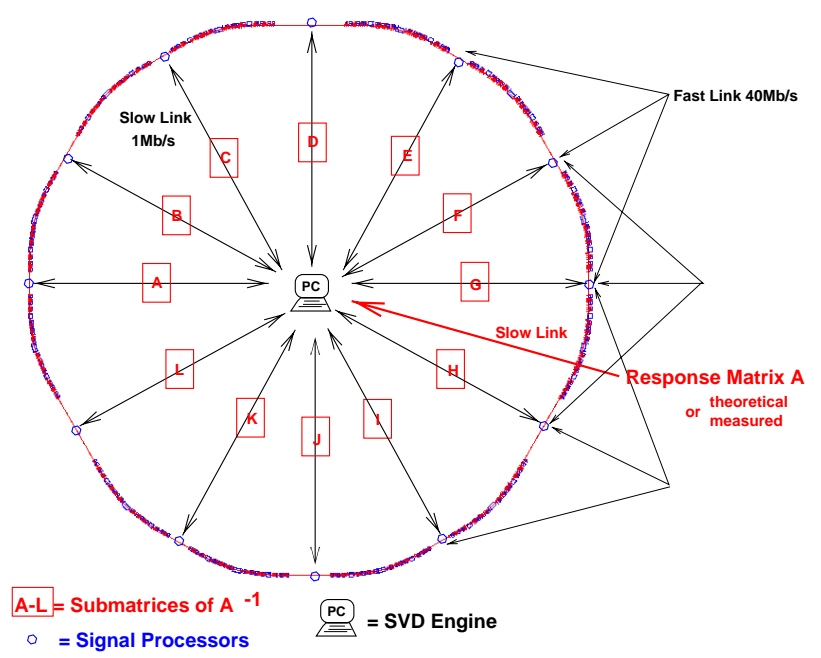

Figure 5: Schematic view of the implementation of the fast orbit feedback

Only if the corrector-monitor set changes the inverted matrix has to be recalculated by the "SVD Engine". This solution requires local intelligence within the sectors. The necessary computing power ist delivered by special signal processor boards which are integral part of the proposed digital monitor system [1]. The links are based on protocol free unidirectional data connections transfering data at a rate of $40 \mathrm{Mb} / \mathrm{sec}$ (see Figure 6 for details). It should be noted that additional monitors at the location of the insertion devices can be easily added to the feedback.

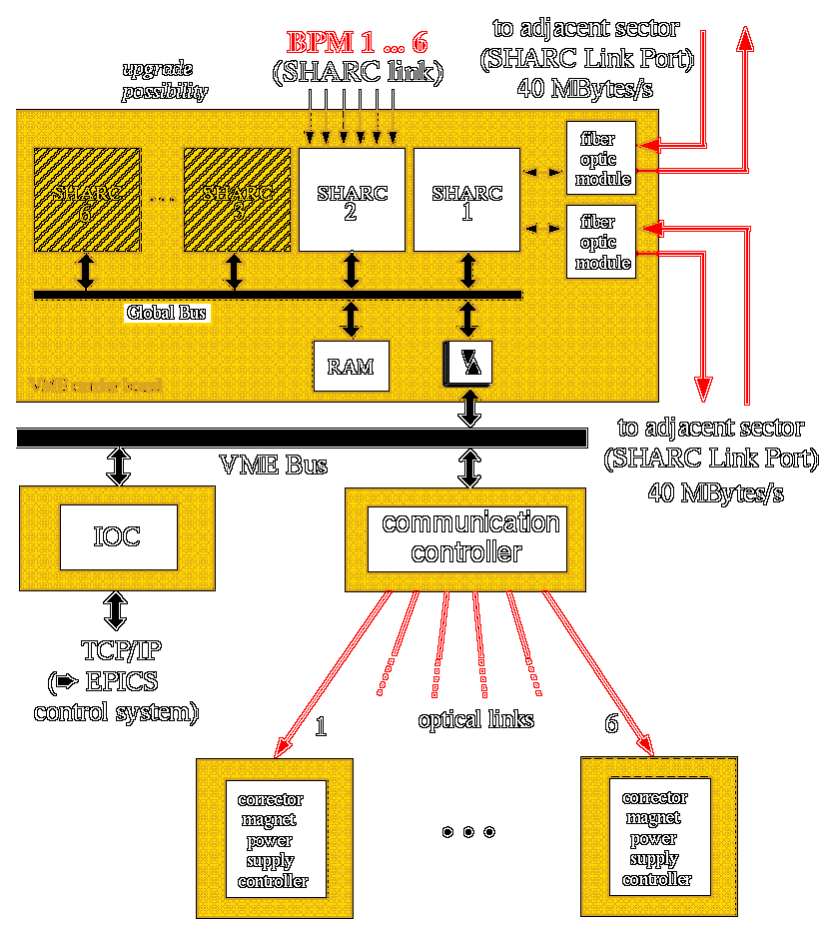

Figure 6: Layout of the fast orbit feedback electronics

\subsection{Simulation}

Based on the previously discussed model a simulation of the feedback loop has been performed based on a PID controller model in order to determine the detailed specifications for the feedback. It has been found that the feedback has to operate at a sampling rate of $4 \mathrm{kHz}$ in order to provide a $-20 \mathrm{~dB}$ orbit noise attenuation at $90 \mathrm{~Hz}$ (see Figure 7). The statistical monitor noise should be not larger than $0.5 \mu \mathrm{m}$ rms. The same holds for the tolerable granularity of the corrector power supplies. For a maximum kick of $1 \mathrm{mrad}$ per corrector the analogue resolution should not exceed 15 ppm in amplitude.

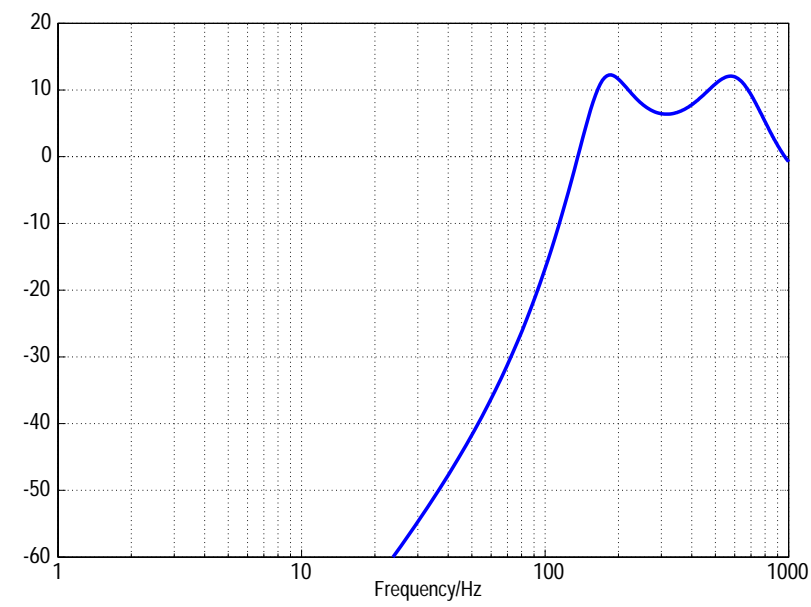

Figure 7: Noise attenuation of the feedback loop at a sampling rate of $4 \mathrm{kHz}$

If these prerequisites are fulfilled an rms orbit stabilization to $1 \mu \mathrm{m}$ and an rms residual angle distortion of $0.1 \mu \mathrm{rad}$ at the location of the insertion devices can be achieved.

\section{REFERENCES}

[1] M. Dehler et al., "BPM System for the Swiss Light Source", Contribution to this Conference.

[2] M. Böge et al., "Studies on Imperfections in the SLS Storage Ring", Contribution to this Conference.

[3] C. Markovits, "Erschütterungsmessungen des Schweiz. Erdbebendienstes am Ort der geplanten Synchrotronlichtquelle SLS auf dem Gelände des PSI West", SLS-TME-TA-19940006, (1994).

[4] P. Wiegand, Private Communication.

[5] W. H. Press et al., "Numerical Recipes", Cambridge Press, (1992). 